\title{
Thermo-diffusion in inertially confined plasmas
}

\author{
Grigory Kagan and Xian-Zhu Tang \\ Theoretical Division, Los Alamos National Laboratory, Los Alamos, NM 87545
}

(Dated: September 15, 2018)

\begin{abstract}
In a plasma of multiple ion species, thermodynamic forces such as pressure and temperature gradients can drive ion species separation via inter-species diffusion. Unlike its neutral mix counterpart, plasma thermo-diffusion is found comparable to, or even much larger than, baro-diffusion. It is shown that such a strong effect is due to the long-range nature of the Coulomb potential, as opposed to short-range interactions in neutral gases. A special composition of the tritium and ${ }^{3} \mathrm{He}$ fuel is identified to have vanishing net diffusion during adiabatic compression, and hence provides an experimental test in which yield degradation is minimized during ICF implosions.
\end{abstract}

In inertial confinement fusion (ICF), the central "hotspot" plasma, assembled by laser-driven spherical implosion 1, contains multiple ion species. Common combinations include low-Z fuel mixtures such as deuterium (D)/tritium (T) and $\mathrm{D}^{3} \mathrm{He}$ with possible addition of high$\mathrm{Z}$ pusher ions, such as carbon or silicon, due to plastic or glass shell mixing [2, 3] into the gas fill. Sometimes high-Z gas dopants such as Ar [4] or Kr [5] are intentionally introduced for diagnostic purposes, as well as to specifically study the pre-mix effects [6, 7]. The powerful thermodynamic forces (e.g. pressure [8 10 and temperature gradients $[9,10]$ ) in an imploding target can drive ion species separation via inter-species diffusion. Observation of the resulting fuel stratification in the DT implosion, which upsets the initially optimal arrangement of equal number densities of $\mathrm{D}$ and $\mathrm{T}$, in experiments [11] and kinetic simulations [12 15] have recently been reported. The targets with high- $Z$ dopants show a particularly strong yield anomaly [6, 7, 16, suggesting that even stronger fuel stratification may take place.

Perhaps the most intriguing physics aspect of interion-species diffusion in a collisional plasma is the role of thermo-diffusion, which, as its name suggests, is driven by the gradients of ion and electron temperatures. The novelty comes through as a sharp contrast to the better-known case of a neutral mixture, where thermo-diffusion is substantially less important than baro-diffusion, though often counteracts it [9]. According to statistical physics, thermo-diffusion strongly depends on the details of the collisional exchange between and within the species [10. Due to the long range nature of Coulomb collisions in plasmas, as opposed to short range collisions between neutral particles, one may expect thermo-diffusion in plasmas and neutral mixtures to be fundamentally different. This difference becomes particularly striking with the observation that plasma baro-diffusion ratio $k_{p}$ is identical to its neutral counterpart [17.

The purpose of this Letter is to present (i) an intu- itively obvious, qualitative analysis that elucidates the enhanced importance of thermo-diffusion in a plasma in comparison with a neutral mixture; (ii) a first-principle derivation of the total diffusive mass flux in a plasma with two ion species. Specifically for (ii), thermo-diffusion ratios associated with the ion and electron temperature gradients, as well as classical diffusion coefficients, are calculated numerically for selected pairs of ion species. In contrast to the neutral mixture case, contributions of thermo- and baro-diffusions are found comparable and reinforcing each other. Even more importantly, when the charge number of one of the ion species becomes large, the former dominates over the latter. The newly developed theory for inter-ion-species diffusion is then employed to investigate whether fuel stratification can be mitigated during ICF implosion. Unlike the prediction of Ref. [18, which implicitly relies on thermo-diffusion being zero [19], suppressing the instantaneous diffusive flux is found possible only in very special mixtures such as $\mathrm{T}^{3} \mathrm{He}$, where electro-diffusion is strong enough to counteract thermo-diffusion.

Inter-ion-species diffusion: Following Landau and Lifshitz [10, the diffusive mass flux in a plasma with two ion species is defined as

$$
\mathbf{i}=\rho_{l}\left(\mathbf{u}_{l}-\mathbf{u}\right),
$$

where $\mathbf{u} \equiv\left(\rho_{l} \mathbf{u}_{l}+\rho_{h} \mathbf{u}_{h}\right) / \rho$ is the center-of-mass velocity with $\rho_{\alpha}, \mathbf{u}_{\alpha}$ the mass density and fluid velocity of species " $\alpha$ ", respectively, and subscripts " $l$ " and " $h$ " refer to the light and heavy ion species. Electron inertia is negligible, so $\rho \equiv \rho_{l}+\rho_{h}$ can be referred to as the plasma density. The diffusive flux governs the evolution of the mass concentration $c \equiv \rho_{l} / \rho$ through 10$]$

$$
\rho \frac{\partial c}{\partial t}+\rho \mathbf{u} \cdot \nabla c+\nabla \cdot \mathbf{i}=0 .
$$

Allowing different electron and ion temperatures $\left(T_{e}\right.$ and $T_{i}$ ), it was recently shown [17] that the diffusive mass flux in a plasma with two ion species take the form 


$$
\mathbf{i}=-\rho D\left(\nabla c+k_{p} \nabla \log p_{i}+\frac{e k_{E}}{T_{i}} \nabla \Phi+k_{T}^{(i)} \nabla \log T_{i}+k_{T}^{(e)} \nabla \log T_{e}\right),
$$

where $p_{i}=p_{l}+p_{h}$ is the ion pressure. The terms on the right side of Eq. (3) represent contributions from, respectively, classical, baro-, electro- and thermo-diffusions. Classical diffusion relaxes concentration perturbations in the absence of externally imposed gradients. Physical picture underlying the other diffusion mechanisms can be understood by considering the relative motion of the light and heavy ion fluids due to external forces. For example, baro-diffusion is due to the $\nabla p_{i}$ force giving a larger acceleration to fluid elements of the light ion species, making $\mathbf{u}_{l}$ and $\mathbf{u}_{h}$ differ. Similarly, electro-diffusion is due to the electric field giving a larger acceleration to the species with higher charge-to-mass ratio. Finally, accelerations of fluid elements of the light and heavy ion species due to the ion-ion and ion-electron thermal forces are generally different as well, giving rise to thermo-diffusions proportional to $\nabla T_{i}$ and $\nabla T_{e}$, respectively. The relative importance of various diffusion mechanisms can therefore be understood by comparing the corresponding forces.

Thermo-diffusion strongly enhanced due to the long range nature of Coulomb interaction - a qualitative demonstration: Before presenting a rigorous first principle calculation of the various diffusion coefficients in Eq. (3), we utilize the intuitive picture of the preceding paragraph to elucidate the importance of thermo-diffusion in plasmas, as opposed to the minor role it plays in neutral gas mixtures. We begin by adapting the qualitative theory of Braginskii 20] to estimate the thermal force in a binary mixture with the collision frequency role explicitly retained.

Consider the two mixture components at a point $x_{0}$ with their net flow velocities equal zero. There are two groups of light particles ("-" and "+") coming from $x_{0}-$ $\lambda$ and $x_{0}+\lambda$, respectively, where $\lambda$ is the mean free path. The thermal force experienced by the light component as a whole arises due to the frictional forces experienced by the two groups, $R_{-}$and $R_{+}$, giving a non-vanishing superposition [20]. These forces are estimated as $R_{-,+} \sim$ $\nu_{l h} m_{l} n_{-,+} v_{-,+}$, where $m_{l}$ is the light particle mass and $\nu_{l h}$ is the characteristic frequency of collisions between the species. $n_{-,+}$and $v_{-,+}$denote the densities and the net flow velocities of the corresponding groups of light particles; for the overall light fluid to remain at rest they must satisfy $n_{-} v_{-}+n_{+} v_{+}=0$. Expanding $T$ about $x_{0}$ and noticing that $n_{-,+} v_{-,+} \sim n_{l} v_{t h}$ with $n_{l}$ and $v_{t h}$ being the number density and thermal velocity of light particles, respectively, the resultant force is estimated as

$$
\begin{aligned}
R_{T} & =R_{+}-R_{-} \sim m_{l} n_{l} v_{t h} \times 2 \lambda \frac{\partial \nu_{l h}}{\partial T} \frac{d T}{d x} \\
& \propto \frac{\partial \ln \nu_{l h}}{\partial \ln T} n_{l} \frac{d T}{d x},
\end{aligned}
$$

where $\lambda=v_{t h} / \nu_{l h}$ is used to obtain the right side.

Next, to contrast thermo-diffusion in neutral mixtures and plasmas with the help of Eq. (4), we consider an ensemble of particles interacting via the potential $\phi(r) \propto 1 / r^{n}$. By balancing the kinetic and potential energies, the closest approach $d$ between particles is found to scale as $d \propto v^{-2 / n}$, where $v \propto \sqrt{T}$ is the characteristic particles' velocities. For the collision crosssection this gives $\sigma \propto d^{2} \propto T^{-2 / n}$ and the collision frequency scaling can be recovered from $\nu_{l h} \propto\langle\sigma v\rangle$, where \langle\rangle denotes the ensemble average. As a result, one finds $\partial \ln \nu_{l h} / \partial \ln T=(n-4) / 2 n$.

In a fully ionized plasma, $n=1$ so the factor on the right side of Eq. (4) is predicted to be $-3 / 2$. Interaction between particles in neutral mixtures is limited to a shorter range. For the purpose of the estimate in simple gases the corresponding $n$ can be placed between 6 and 12 [21], predicting the factor on the right side of Eq. (4) to lie between $1 / 6$ and $1 / 3$. Hence, while in neutral mixtures thermo-diffusion should indeed be substantially less significant than baro-diffusion, the two should be comparable in plasmas with multiple ion species. Equally important, the qualitative analysis also shows that the thermal force by the heavy ion species on the light one, $R_{T}^{l h}$, is in the direction opposite to the temperature gradient $\left(\partial \ln \nu_{l h} / \partial \ln T<0\right)$. Obviously, $R_{T}^{h l}=-R_{T}^{l h}$ and therefore, as far as the relative motion of the two species is concerned, the ion-ion thermal and $\nabla p_{i}$ forces act together. It is thus predicted that, unlike the neutral mixture case $\left(\partial \ln \nu_{l h} / \partial \ln T>0\right)$, thermo-diffusion with $\nabla T_{i}$ reinforces baro-diffusion in plasmas.

The thermal forces exerted onto the two ion species by electrons are aligned and their combined effect on these species' relative motion is not possible to predict with the above simplified analysis. As the rigorous calculation will show, $k_{T}^{(e)}$ and $k_{p}$ may have opposite signs, but in such a case $\left|k_{T}^{(e)}\right|$ is noticeably less than $\left|k_{T}^{(i)}\right|$ and the overall thermo-diffusion should reinforce baro-diffusion still.

Quantifying thermo-diffusion via first-principle calculation: We now proceed with first principle evaluation of the diffusion coefficients in Eq. (3). The total collisional force exerted on species $\alpha$ by other species is known to have the form [22, 23.

$$
\mathbf{R}_{\alpha}=-\sum_{\beta}\left[A_{\alpha \beta} \mu_{\alpha \beta} n_{\alpha} \nu_{\alpha \beta}\left(\mathbf{u}_{\alpha}-\mathbf{u}_{\beta}\right)+B_{\alpha \beta} n_{\beta} \nabla T_{\beta}\right],
$$

where $T_{\alpha}$ and $m_{\alpha}$ are, respectively, the temperature and elementary mass of species $\alpha ; \mu_{\alpha \beta} \equiv m_{\alpha} m_{\beta} /\left(m_{\alpha}+m_{\beta}\right)$ is the reduced mass and $\nu_{\alpha \beta}$ stands for the frequency of collisions between species $\alpha$ and $\beta$. Subscripts " $\alpha$ " and " $\beta$ " can take values "l", " $h$ " and " $e$ " to denote light ion, 
heavy ion and electron species, respectively. The first term on the right side represents dynamic friction, and $A_{\alpha \beta}=A_{\beta \alpha}$ due to momentum conservation. For realistic currents the effect of ion-electron friction on mass diffusion can be neglected $\left(A_{h e}, A_{l e} \approx 0\right)$ [17. The second term on the right side represents thermal forces. The ion-electron thermal force is governed solely by the electron temperature gradient $\left(B_{e l}, B_{e h} \approx 0\right)$, because the thermal speed of electrons is much greater than that of ions; both $B_{l l}$ and $B_{l h}\left(B_{h h}\right.$ and $\left.B_{h l}\right)$ contribute to the ion-ion thermal force.

Utilizing Eq. (5) to write the momentum conservation equations for the two ion species and imposing the shortmean-free-path ordering one can obtain the diffusive flux in the form (3) from definition (1) with [17.

$$
\begin{aligned}
D= & \frac{\rho T_{i}}{A_{l h} \mu_{l h} n_{l} \nu_{l h}} \times \frac{c(1-c)}{c m_{h}+(1-c) m_{l}}, \\
k_{p}= & c(1-c)\left(m_{h}-m_{l}\right)\left(\frac{c}{m_{l}}+\frac{1-c}{m_{h}}\right), \\
k_{E}= & m_{l} m_{h} c(1-c)\left(\frac{c}{m_{l}}+\frac{1-c}{m_{h}}\right)\left(\frac{Z_{l}}{m_{l}}-\frac{Z_{h}}{m_{h}}\right), \\
k_{T}^{(i)}= & m_{l} m_{h}\left(\frac{c}{m_{l}}+\frac{1-c}{m_{h}}\right)\left[\frac{c B_{l l}}{m_{l}}+\frac{(1-c) B_{l h}}{m_{h}}\right], \\
k_{T}^{(e)}= & m_{l} m_{h}\left(\frac{c}{m_{l}}+\frac{1-c}{m_{h}}\right)\left[\frac{c Z_{l}}{m_{l}}+\frac{(1-c) Z_{h}}{m_{h}}\right] \times \\
& {\left[(1-c) B_{l e}-c B_{h e}\right] \frac{T_{e}}{T_{i}}, }
\end{aligned}
$$

where $Z_{\alpha}$ denotes the charge number of species $\alpha$. Importantly, for comparable $m_{l}$ and $m_{h}$ the time of equilibration within an ion species is about the same as that of equilibration between the species, making $T_{l} \approx T_{h} \equiv T_{i}$. Equilibration between electrons and ions requires a much longer time, so distinction between $T_{i}$ and $T_{e}$ should generally be kept. This said, the ion-ion collision frequency can be written as

$$
\nu_{l h}=\frac{4(2 \pi)^{1 / 2}}{3} \frac{n_{h}}{\mu_{l h}^{1 / 2} T^{3 / 2}}\left(\frac{Z_{l} Z_{h} e^{2}}{4 \pi \varepsilon_{0}}\right)^{2} \ln \Lambda,
$$

where $\ln \Lambda$ is the Coulomb logarithm.

It can be observed that the baro- and electro-diffusion ratios $k_{p}$ and $k_{E}$ are obtained without invoking a kinetic calculation, i.e. they are thermodynamic quantities [10, 17. The classical diffusion coefficient $D$ involves the transport coefficient $A_{l h}$ defining dynamic friction between the ion species according to Eq. (5). This has a clear physical interpretation: it is the friction that prevents the species from running apart, so larger $A_{l h}$ should result in smaller $D$, as it is indeed predicted by Eq. (6). Thermo-diffusion ratios $k_{T}^{(i)}$ and $k_{T}^{(e)}$ involve $B_{l l}$ and $B_{l h}$ and $B_{l e}$ and $B_{h e}$, respectively. According to Eq. (5), these coefficients govern the ion-ion and ion-electron thermal forces, respectively; Eqs. (9)-(10) therefore agree with the intuitive picture presented earlier in this Letter. The fact that $A_{\alpha \beta}$ and $B_{\alpha \beta}$ enter
Eqs. 64, (9) and 10 suggests that $D, k_{T}^{(i)}$ and $k_{T}^{(e)}$ are non-thermodynamic; deviation of the species' distribution functions from Maxwellian needs to be accounted for when evaluating these coefficients.

Hence, we are led to conduct a kinetic calculation to obtain $A_{\alpha \beta}$ and $B_{\alpha \beta}$. To this end, the monograph by Zhdanov 22] turns out to be instrumental. Ref. 22 employs the Grad $21 \mathrm{~N}$ moment method, where kinetic equation for each species is replaced with a set of moment equations of this species' distribution function, which is truncated to keep the first 21 moments. Importantly, when evaluating transport in neutral gases, keeping 13 moments per species is sufficient, whereas to achieve satisfactory precision of transport calculation in unmagnetized plasmas (or longitudinal transport calculation in magnetized plasmas) keeping 21 moments is necessary. This is a mathematical manifestation of the peculiarity of Coulomb collisions, as compared to the shorter range collisions in neutral gases, that was explained in physical terms earlier in this Letter.

By solving the short-mean-free-path version of the moment equations of Ref. [22, $A_{\alpha \beta}$ and $B_{\alpha \beta}$ as functions of the species concentrations, elementary masses and charge numbers can be obtained. When $m_{l}$ and $m_{h}$ are comparable the analytical expression for $A_{l h}, B_{l l}$ and $B_{l h}$ becomes cumbersome, so we numerically evaluate dependence of these coefficients on the light species mass concentration $c$ for selected pairs of ion species. The dynamic friction coefficient $A_{l h}$ as a function of $c$ is plotted in Fig. 1. Using numerically obtained $B_{l l}(c)$ and $B_{l h}(c)$ along with Eq. $(9), k_{T}^{(i)}(c)$ is evaluated and plotted in Fig. 2,

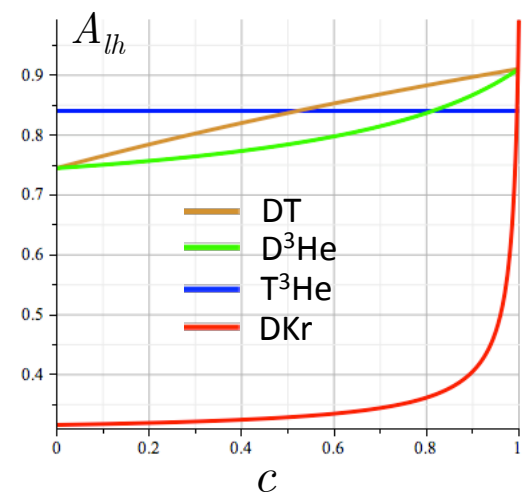

FIG. 1. The dynamic friction coefficient $A_{l h}$ as a function of $c$.

Since $m_{e} \ll m_{l, h}$ moment equations for electrons can be separated from those for the two ion species [22. This allows tractable expressions for $B_{l e}$ and $B_{h e}$, which, upon inserting into Eq. 10, give

$k_{T}^{(e)}=-m_{l} m_{h} c(1-c)\left(\frac{c}{m_{l}}+\frac{1-c}{m_{h}}\right)\left(\frac{Z_{l}^{2}}{m_{l}}-\frac{Z_{h}^{2}}{m_{h}}\right) \frac{T_{e}}{T_{i}} \frac{B_{e e}}{Z_{\text {eff }}}$, 
where $Z_{\text {eff }} \equiv\left(n_{l} Z_{l}^{2}+n_{h} Z_{h}^{2}\right) / n_{e}$ is the effective ion charge number, $n_{\alpha}$ the number density of species $\alpha$, and

$$
B_{e e} \approx \frac{0.47+0.94 Z_{\text {eff }}^{-1}}{0.31+1.20 Z_{\text {eff }}^{-1}+0.41 Z_{\text {eff }}^{-2}} .
$$

Eqs. 12)- 13) give the $k_{T}^{(e)}(c)$ dependence for any given $m_{l, h}$ and $Z_{l, h}$; this dependence is plotted in Fig. 2 for the pairs of ion species, for which $k_{T}^{(i)}(c)$ is evaluated numerically.
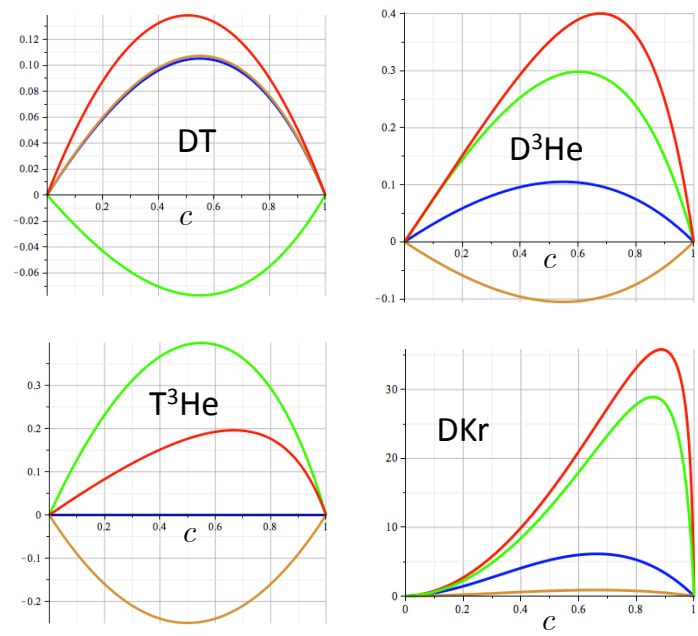

$-k_{p}-k_{E}-k_{T}^{(i)}-k_{T}^{(e)}$

FIG. 2. Diffusion ratios $k_{p}, k_{E}, k_{T}^{(i)}$ and $k_{T}^{(e)}$ as functions of c.

Eq. (3), along with Eqs. (7), (8) and (12) and data in Figs. 1 and 2, provide a first-principle based description of mass diffusion in a plasma with two ion species. With this result in hand, we are in a position to evaluate the practical implications of this phenomenon in ICF plasmas.

It should first be observed in Fig. 1 that when ion masses are close, kinetic effects do not introduce a substantial $c$ dependence in $D$. In particular, for the $\mathrm{T}^{3} \mathrm{He}$ mix, where $m_{l}=m_{h}, A_{l h}(c)$ is perfectly flat. However, as the mass ratio becomes larger, $A_{l h}$ may vary with concentration by a noticeable factor, which is about three for the DKr mix $\left(m_{h} / m_{l} \approx 42\right)$.

Second, in agreement with the qualitative analysis presented earlier in this Letter, thermo-diffusion due to ion temperature gradient always reinforces baro-diffusion. In contrast with the neutral mixture theory, baro-diffusion by no means dominates over thermo-diffusion. Instead, for low- $Z$ mixtures with comparable masses $k_{T}^{(i)}$ is slightly larger than $k_{p}$. For the DT mix, $k_{T}^{(e)}$ is negative, but small and is unlikely to substantially affect the mass flux.

Finally, in capsules doped with high- $Z$ impurities such as $\mathrm{Kr}$, it is thermo-diffusion, with both $\nabla T_{i}$ and $\nabla T_{e}$, that dominates over baro-diffusion!
Mitigating fuel stratification: In principle, mitigating the negative consequence of inter-ion-species diffusion for ICF does not necessarily require suppressing the diffusive flux at every point in time in space throughout the implosion. One could attempt to balance the concentration perturbations at different stages of the implosion so that by the burn time the concentration breakdown becomes the optimal one. However, this path requires a very careful timing of the implosion and the diffusion effect may be difficult to untangle from other potential sources of discrepancy between single-fluid simulations and the experiment. For a more robust verification of the effect, it is desirable to find a case with $\mathbf{i} \equiv 0$ and $c=$ const.

Considering the adiabatic case with $T_{e}=T_{i} \equiv T$ and setting the right side of Eq. (3) equal zero gives a solubility constraint in terms of the diffusion ratios

$$
1-\gamma=\frac{k_{p}+k_{E}}{k_{p}+k_{E}+k_{T}^{(i)}+k_{T}^{(e)}+B_{e e} k_{E}} .
$$

With the results of Fig. 2 this constraint, in turn, gives an equation for $c$, for which the diffusion vanishes. Using the numerically obtained dependencies of the diffusion ratios on $c$ and taking $\gamma=5 / 3$, we find that $\mathbf{i} \equiv 0$ is not possible to achieve for the DT mix. In the $\mathrm{D}^{3} \mathrm{He}$ mix, electro-diffusion acts in the favorable direction, but is still unable to cancel the combined effect of the baroand thermo- diffusions. However, the $\mathrm{D}^{3} \mathrm{He}$ case suggests that the desirable regime may be realizable for the pair of species with larger charge-to-mass difference, and therefore stronger electro-diffusion, such as $\mathrm{T}^{3} \mathrm{He}$. This is confirmed by a numerical calculation, which shows that for the $\mathrm{T}^{3} \mathrm{He}$ diffusion ratios Eq. (14) does have a solution at $c \approx 0.89$.

In conclusion, first-principle derivation of the diffusive mass flux in a plasma with two ion species reveals the paramount importance of thermo-diffusion. This is fundamentally due to the long range nature of Coulomb potentials, in sharp contrast to short range collisions in neutral gases. Specifically, thermo-diffusion is found to contribute to species separation as much as baro-diffusion in low-Z ion mixtures and dominate over baro-diffusion in plasmas with high-Z dopants. Since the thermo-diffusion ratios are much greater than unity in the presence of a high-Z ion species, thermo-diffusion has the potential to drive significant species separation when temperature gradient is large and may contribute to the aggravated yield anomaly in the doped capsule experiments. We also show that diffusionless implosion is possible in $\mathrm{T}^{3} \mathrm{He}$ capsule with the concentration of $\mathrm{T}$ about $c_{*} \approx 0.89$. Assuming that other mechanisms potentially responsible for the yield anomaly are less sensitive to $c$, the role of the ion concentration diffusion on the performance of ICF capsules can thus be studied by performing implosions of $\mathrm{T}^{3} \mathrm{He}$ filled targets with various concentrations 
of T. The discrepancy between the experimentally observed yield of the reaction products with that predicted by standard single-fluid rad-hydro codes should then experience a minimum for $c \approx c_{*}$.

The authors are indebted to the members of the MIT HEDP group for their many insights into experimental implications of the inter-ion-species diffusion. They also wish to thank F. J. Wysocki, G.A. Kyrala, Y.H. Kim and H.W. Herrmann of LANL and P. Helander of IPPGreifswald for useful discussions. This work was supported by the Laboratory Directed Research and Development (LDRD) program of LANL.

[1] J. Nuckolls, L. Wood, A. Thiessen, and G. Zimmerman, Nature (London) 239, 139 (1972).

[2] C. K. Li, F. H. Sguin, J. A. Frenje, S. Kurebayashi, R. D. Petrasso, D. D. Meyerhofer, J. M. Soures et al, Phys. Rev. Lett. 89, 165002 (2002).

[3] D. C. Wilson, P. S. Ebey, T. C. Sangster, W. T. Shmayda, and R. A. Lerche, Phys. Plasmas 18, 112707 (2011).

[4] S. P. Regan et al., Phys. Plasmas, 9, 1357 (2002).

[5] B. Yaakobi, R. Epstein, C. F. Hooper, Jr., D. A. Haynes, Jr, and Q. Su, J. of X-ray Sci. and Tech. 6, 172 (1996).

[6] D. C. Wilson, G. A. Kyrala, J. F. Benage Jr, F. J. Wysocki, M. A. Gunderson, W. J. Garbett, V. Yu Glebov et al, J. Phys.: Conf. Ser. 112, 022015 (2008).

[7] W. J. Garbett, S. James, G. A. Kyrala, D. C. Wilson, J. F. Benage, F. J. Wysocki, M. A. Gunderson, J. Frenje, R. Petrasso, V. Yu. Glebov, and B. Yaakobi, J. Phys.: Conf. Ser. 112, 022016 (2008).

[8] P. Amendt, O. L. Landen, H. F. Robey, C. K. Li and R. D. Petrasso, Phys. Rev. Lett. 105, 115005 (2010).
[9] Ya. B. Zeldovich and Yu. P. Raizer, Physics of Shock Waves and High-Temperature Hydrodynamic Phenomena (Dover, Mineola, New York, 2002).

[10] L. D. Landau and E.M. Lifshitz, Fluid Mechanics (Oxford, Pergamon, 1958).

[11] D. T. Casey, J. A. Frenje, M. G. Johnson, M. J. E. Manuel, H. G. Rinderknecht, N. Sinenian, F. H. Séguin, and C. K. Li, R. D. Petrasso et al, Phys. Rev. Lett. 108, 075002 (2012).

[12] O. Larroche, Phys. Plasmas 19, 122706 (2012).

[13] C. Bellei, P. A. Amendt, S. C. Wilks, M. G. Haines, D. T. Casey, C.K. Li, R. Petrasso, and D. R. Welch, Phys. Plasmas 20, 012701 (2013).

[14] O. Larroche, Phys. Plasmas 20, 044701 (2013).

[15] C. Bellei, P. A. Amendt, S. C. Wilks, M. G. Haines, D. T. Casey, C.K. Li, R. Petrasso, and D. R. Welch, Phys. Plasmas 20, 044702 (2013).

[16] E. S. Dodd, J. F. Benage, G. A. Kyrala, D. C. Wilson, F. J. Wysocki, W. Seka, V. Yu Glebov, C. Stoeckl, and J. A. Frenje, Phys. Plasmas 19, 042703 (2012).

[17] G. Kagan and X.Z. Tang, Phys. Plasmas 19, 082709 (2012).

[18] P. Amendt, C. Bellei and S.C. Wilks, Phys. Rev. Lett. 109, 075002 (2012).

[19] P. Amendt, C. Bellei and S.C. Wilks, Phys. Rev. Lett. 109, 269502 (2012).

[20] S. I. Braginskii, Reviews of Plasma Physics 1, 205 (1965).

[21] J. O. Hirschfelder, C. F. Curtiss and R. B. Bird, Molecular theory of gases and liquids (Vol. 26) (New York, Wiley, 1954).

[22] V. M. Zhdanov, Transport Processes in Multicomponent Plasma, Taylor and Francis, New York, (2002).

[23] S. Hirshman and D. Sigmar, Nucl. Fusion 21, 1079 (1981). 